\title{
Cold Air Drainage on a Forested Mountain Slope ${ }^{1}$
}

\author{
James D. BERGEN \\ Rocky Mountain Forest and Range Experiment Station, Forest Service, Fort Collins, Colo. ${ }^{2}$
}

(Manuscript received 23 June 1969, in revised form 2 September 1969)

\begin{abstract}
The nocturnal cold airflow on a small forested slope was studied in an attempt to relate the volume and velocity of flow to the net radiation balance of the slope.

The hillside, with an approximately $30 \%$ grade, was about $400 \mathrm{~m}$ long and covered with an uneven-aged spruce-fir stand of about $20 \mathrm{~m}$ height. The location was near Fraser, Colo., at an altitude of $3000 \mathrm{~m}$.

The vertical profiles of wind speed and air temperature were measured at various points on a transect approximating a streamline segment down the hill for several nights. Net radiation was measured at the base of the transect. Canopy closure and tree heights and diameters were measured on sample plots along the transect.

The vertical profiles of potential temperature deficit and wind speed at the various stations show approximate similarity when scaled by the height of the lowest inversion and by the average velocities and potential temperature deficits below that inversion. The latter varies in a nearly linear manner down the slope, and the inversion heights appear to be relatively constant over the range of net radiation rates observed.

Assuming a corresponding analytical similarity for the equations of motion and the sensible heat balance, the last two phenomena are found to imply that

$$
U_{m} \propto \sqrt{\Delta \theta}, \quad \Delta \theta \propto R_{0}, \quad U_{m} / \sqrt{\gamma} \propto x,
$$

where $U_{m}$ is the average velocity indicated above, $\Delta \theta$ the potential temperature drop down the slope, $\gamma$ the sine of the angle of the slope to the horizontal along the streamline, $x$ the downslope distance from the virtual origin of the flow measured along the streamline, and $R_{0}$ the average net radiation loss on the slope.

The data appear to confirm these results.

The constants of proportionality calculated from the topographic contours, the estimated foliage distribution, and the scaled profiles of wind speed and potential temperature deficit are in fair agreement with observations.
\end{abstract}

\section{Introduction}

Steep topography and frequent clear, dry nights make nocturnal density flows a major determinant for local climate in the Colorado Rockies. This is particularly true in the Middle Park area, where the lowest regularly observed temperatures in the Continental United States are associated with energetic circulations between the surrounding wooded and alpine slopes and the open valley of the Fraser River.

These circulations were first studied by Ives (1939) as the cause of river fog phenomena in the Fraser valley. At the valley bottom, the wind and temperature field is a complex resultant of the cold airflows from a number of slopes that vary widely in contour and forest cover, with the overall synoptic scale flow over the Park. The study described in this paper was an attempt to analyze one small but fundamental element of this circulation: the downslope flow of cold air from an isolated forested hillside subject to relatively simple geometrical restraints on the flow divergence and the distribution of

\footnotetext{
1 Presented at Eighth Natl. Conf. on Agric. Meteor., Amer. Meteor. Soc., Ottawa, 21-23 May 1968.

"Central headquarters at Fort Collins, in cooperation with Colorado Statce University.
}

radiation loss. The immediate objective was a relation between the velocity and temperature of the nocturnal cold airflow off this hillside and its net radiation balance.

It was obvious from the complexity of the problem that such a relationship would be very restricted in application. No generally satisfactory models have been offered for turbulent exchange processes either in uniform flow with stable stratification, or for unstratified flow in a forest canopy, and the density flow on the hillside involves the difficulties of both problems, in addition to those raised by its nonuniformity. However, in view of the importance of such flows in ecology and agriculture, even the very tentative conclusions that can be reached seemed to warrant consideration of the problem. The results may also be of interest to those concerned with air pollution in such environments, since the observed concentration of the flow in the levels including the forest canopy implies that the foliage may radically affect the concentration of particulate pollution downstream from such slopes.

\section{Topography}

The site of the observations is a forest-covered slope in the Fraser Experimental Forest near Fraser, Colo., 
at an elevation of about $3000 \mathrm{~m}$. The topography of the site is shown in the contour map of Fig. 1. The slope is at the northeastern end of a long ridge separated from adjacent slopes by stream gullies on either side, and facing in to the relatively level expanse of Middle Park.

If the streamlines of the surface airflow on clear nights are assumed to be normal to the height contour lines, the resulting streamline pattern is that shown in Fig. 1. The position of the measurement stations are also shown in Fig. 1. As may be seen, the stations are approximately located along a streamline. The streamlines bordering the transect appear to originate at a center of divergence $\mathrm{C}$ located about halfway up the hill. This point was used as the origin of coordinatesthe effective beginning - of the drainage flow on the experimental hillside.

A topographic divergence index may be defined as the fractional increase in the area $A^{\prime \prime}$ of an air parcel projected normal to the slope, per unit distance of motion $d x$ along the streamline originating at $\mathrm{C}$, i.e., $\left(d A^{\prime \prime} /\right.$ $d x) A^{\prime \prime-1}$. This quantity was calculated with finite differences for the streamline along the transect at the station positions and one intermediate point. A plot of the results shown in Fig. 2 indicates that it varies approximately as $x^{-1}$, as would be the case for flow down a conical section.

Since the slope faced a relatively open valley extending about $15 \mathrm{mi}$ to the Continental Divide, the effect of the downslope variation of the relative view factors of sky and horizon was neglected in the subsequent analysis.

\section{Foliage}

The trees on the slope are an uneven-aged mixture of spruce, fir and pine (typical of this altitude in the central Rockies) with a maximum height of about $20 \mathrm{~m}$.

\section{a. Distribution of radiating surface}

A plausible argument may be made (Reifsnyder and Lull, 1963) that the average view factor for a foliage surface relative to the sky at a level $z$ above the slope is unity less a term proportional to the average amount of foliage surface above that level. If the net radiation loss $R_{\mathfrak{0}}$ per unit area with a sky view factor of unity. is approximated by that for a blackbody at the average slope temperature, then the average net radiation loss $R$ per unit volume from the foliage surfaces may be written as

$$
R / R_{0}=\rho_{z} \alpha^{\prime}\left(1-Q \int_{z}^{\infty} \rho_{z} d z\right)
$$

where $\rho_{z}$ is the average weight concentration of foliage per unit volume of the canopy, $\alpha^{\prime}$ the ratio of foliage surface to foliage weight, and $Q$ a constant.

The variation of foliage concentration $\rho_{z}$ with height on the experimental slope was estimated from a survey

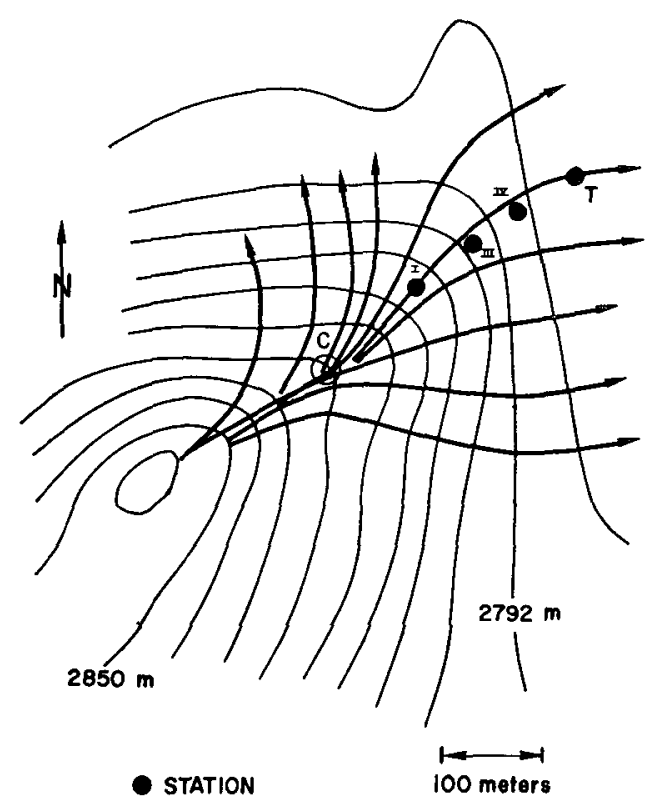

FIG. 1. Estimated streamlines.

of stem diameters, height, and canopy diameters on two sample plots. The stem diameter and the live crown length were used to estimate the total weight of foliage for each tree by means of available empirical relations (Storey et al., 1955). The total foliage weight for each tree crown was assumed to be distributed with height in the same proportions as the volume of a cone with the same base diameter and length as the tree crown.

\section{b. Canopy cover}

Measurements with a mirror densiometer indicate that the average canopy cover $C^{\prime}$ more than a meter

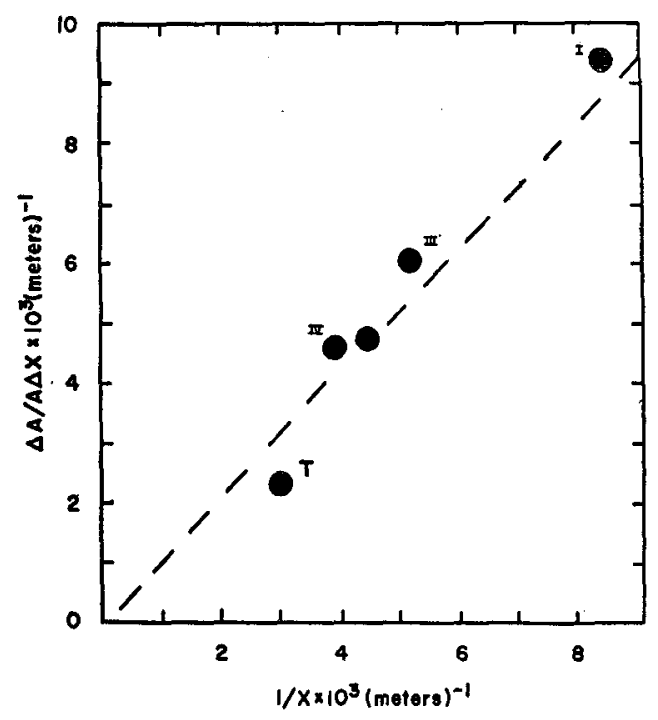

FIG. 2. Variation of topographic divergence along transect. 
TABLE 1. Levels and locations of wind speed and temperature measurements.

\begin{tabular}{|c|c|c|c|c|}
\hline Station & $\begin{array}{l}\text { Wind speed } \\
\text { levels } \\
\text { (m) }\end{array}$ & $\begin{array}{l}\text { Air temperature } \\
\text { levels } \\
(\mathrm{m})\end{array}$ & $\begin{array}{l}\text { Wind speed } \\
\text { levels } \\
\text { (m) }\end{array}$ & $\begin{array}{l}\text { Air temperature } \\
\text { levels } \\
\text { (m) }\end{array}$ \\
\hline I & & $\begin{array}{l}6.0,7.0,9.0,12.0 \\
0.7^{\mathrm{d}}\end{array}$ & $0.5,1.8,3.9,14.0$ & $0.5,1.8,3.9,14.0$ \\
\hline III & $\begin{array}{l}3.1^{\mathrm{o}}, 6.1^{\mathrm{o}}, 9.4^{\mathrm{c}}, 11.0^{\mathrm{c}}, \\
16.0^{\mathrm{a}}\end{array}$ & $0.7^{\mathrm{d}}, 10.0,12.0$ & $\begin{array}{l}0.4,1.7,3.1,5.9 \\
\quad 7.4,8.7,10.2,14.6\end{array}$ & $\begin{array}{l}0.4,1.7,3.1,5.9,7.4,8.7 \\
\quad 7.4,8.7,10.2,14.6\end{array}$ \\
\hline IV & & $0.7^{\mathrm{d}}, 2.3,4.3,6.0,8.3$ & $0.3,1.5,3.0$ & $0.3,1.5,3.0$ \\
\hline Tower & $20.0^{\mathrm{c}}$ & $1.3^{\mathrm{a}}, 2.5^{\mathrm{a}}, 5.0^{\mathrm{a}}, 10.0^{\mathrm{a}}$ & $3.0^{\mathrm{b}}, 5.9,11.4,20.0$ & $\begin{array}{r}1.3,2.5,5.0,5.9 \\
10.0,11.4,20.0\end{array}$ \\
\hline Screen & & $3.0^{\mathrm{d}}$ & & $3.0^{\mathrm{d}}$ \\
\hline
\end{tabular}

a Thermocouples.

b Thermocouple anemometer.

c Cup anemometers.

d Hygrothermograph.

from a tree trunk is $37 \%$, and that the average cover at $\frac{1}{2}$ crown radius from the trunk of a tree is $77 \%$ on the sample plots.

The latter measurement was used to evaluate $Q$ at $z=1 \mathrm{~m}$ in the equation above. The value of $\alpha^{\prime}$ was assumed to be $11 \mathrm{~m}^{2} \mathrm{~kg}^{-1}$ (Storey et al., 1955). The consequent calculated variation of $R / R_{0}$ is shown in Fig. 3 . The power law $R / R_{0}=E / z$, where $E=0.05$, appeared to yield a fair fit to the curve for heights at which the amount of foliage is significant; the relation is assumed to apply only at heights $>1 \mathrm{~cm}$ from the slope surface.

The estimated stand characteristics used in the analysis of the flow, in addition to the distribution of radiating surface, were the average diameter $D$ of the intertree space and the percentage of the slope area which is not under trees.

The latter was estimated at $35 \%$ on the sample plots, and if the stand were hypothetically replaced by a uni-

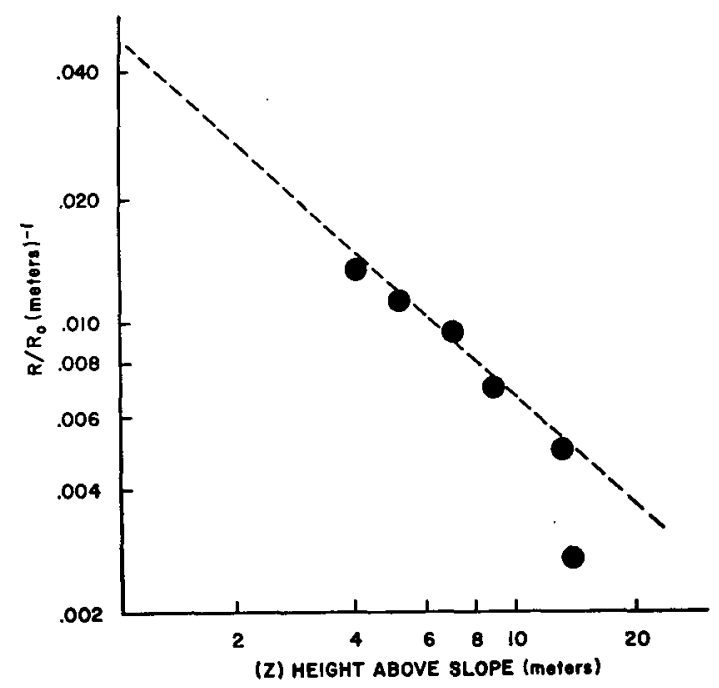

FIG. 3. Variation of foliage radiating surface with height. form, regularly spaced stand of the same total projected canopy area, the average distance between tree canopies at mid-crown level would be about $1 \mathrm{~m}$.

It is apparent, in view of the sampling problems involved and the assumptions made in the preceding calculations, that the values of $E, D$, and the canopy cover $C^{\prime}$ of the inter-tree space arrived at are very approximate estimates.

\section{Observations}

Measurements of wind speed and air temperature on the hillside from about 1800 to 0600 local standard (LST) were made for a total of 14 nights in 1962, 1964 and 1966, when the $20 \mathrm{~m}$ wind speed at the tower was less than $0.3 \mathrm{~m} \mathrm{sec}^{-1}$. In 1962, air temperature at 0.3 , 1.7 and $5.0 \mathrm{~m}$ were measured about $10 \mathrm{~m}$ on each of the transects at stations I, III and IV (Fig. 1). Single stations were located on the transect half way between I and III, and III and IV. All profile stations had an unimpeded fetch upslope for at least $5 \mathrm{~m}$.

Air temperature was measured with bead thermistors in radiation shields extended across the slope on crossarms. A hygrothermograph was mounted in a screen at $3.0 \mathrm{~m}$ height $100 \mathrm{~m}$ east of the tower station. The tower at the base of the slope ( $\mathrm{T}$ in Fig. 1) was equipped with shielded thermocouples for air temperature measure. ments at the 1.25, 2.5, 10.0,15.0, and $20.0 \mathrm{~m}$ levels, and a recording cup anemometer and vane at the $20 \mathrm{~m}$ level.

In 1962, wind speeds were measured only at station IV, with a set of sensitive cup anemometers mounted on a mast at levels of $20,40,80$ and $160 \mathrm{~cm}$; counters were read at 10 -min intervals. Smoke grenades periodically ignited during observations indicated an inversion at $10 \mathrm{~m}$ at $\mathrm{T}$. The location and height of the wind speed and air temperature measurements made in the more extensive observations of 1964 and 1966 are shown in Table 1. For these measurements, a ventilated radiometer shielded to indicate sky radiation was operated at 
the tower. Air temperature and radiometer output were recorded at 12-min intervals. Microbarographs were placed on the hillside stations. Sensitive cup anemometers and a heated thermocouple anemometer were used in 1964. In 1966, heated thermistor anemometers were used, as described in detail in a previous paper (Bergen, 1967, pp. 24-29). Their output was a good approximation to a moving $10-\mathrm{min}$ average of the wind speed. The associated readout circuit registered the simultaneous values of this average wind speed for each of the various points on the hillside.

\section{Survey of data}

\section{a. Temperature}

The vertical profile of the average air temperature and the associated wind speed profile over a 2-hr interval are illustrated in Fig. 4 for the 2400-0200 period on June 1966, for the mid-slope station. These profiles are typical of those found at all the stations. The main features of the temperature profile are a region of positive gradient near the surface of the slope, an inversion at some height $L$ above the slope, and a subsequent temperature minimum, in turn followed by an increase in temperature with height. A second inversion is located above the span of the instrument array, very probably at about $30 \mathrm{~m}$ above the slope in the case of the given profile. The figure of $30 \mathrm{~m}$ is a rough estimate from the temperature drop over the profile from 1800 LST of the same day, and the trace of the microbarograph located at the station. While the intensity of the lowest inversion increases through the night, its height at a particular station remains constant through the night and between nights.

The spacing of instruments at the mid-slope station III and at the tower for the 1966 observations was such that the inversion height $L$ can be determined within approximately $1 \mathrm{~m}$; however, the measurements for station I for all periods and those of 1964 for all stations were too widely spaced to allow any comparable resolution. The value of $L$ used for the tower and station III was the average of the two consecutive heights of the height interval more than a meter above the surface showing the maximum temperature rise with height. For the nights of 14-16 June 1966, $L$ was $11 \mathrm{~m}$ at the tower station and $7 \mathrm{~m}$ at the mid-slope station. For the nights of 11 and 12 June $L$ decreased to $10 \mathrm{~m}$ at the tower and dropped perceptibly at the mid-slope station.

The variation of sensible heat content along the transect is more accurately indicated by the "potential temperature" $\theta$ of the air. For convenience in the analysis, "potential temperature" here will refer to the temperature of a parcel brought along a dry adiabatic to the standard pressure at the altitude of the virtual origin $\mathrm{C}$ rather than to the conventional $1000 \mathrm{mb}$. The temperatures at stations I, III, and the tower must be decreased by $0.3,0.5,0.8 \mathrm{C}$, respectively, to compute the downslope variation in $\theta$ as so defined.
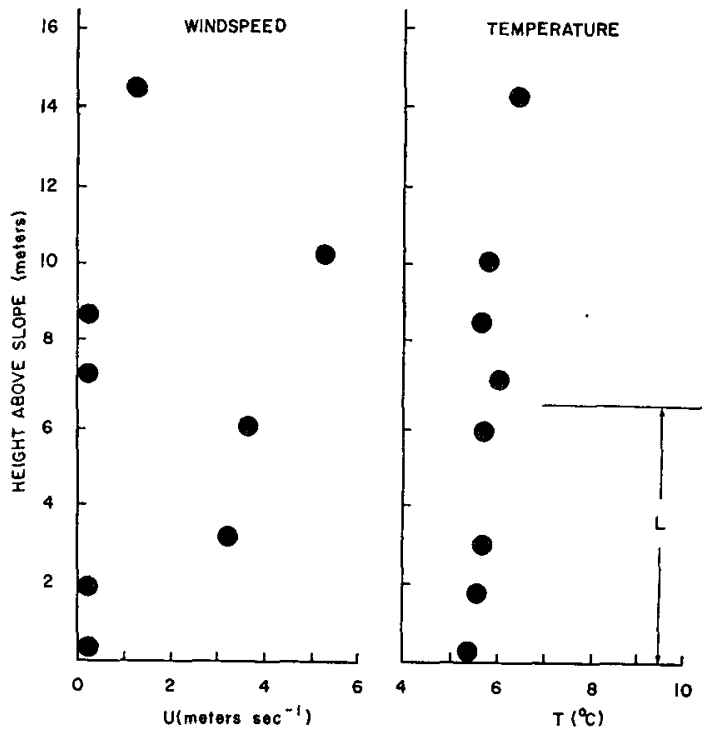

FIG. 4. Wind speed and temperature profles, station III, 24000200 LST 15 June 1966.

Since the choice of the dry adiabatic correction implies that the air is above its dew point at all points on the slope, it should be noted that periodic inspections made during the observations showed no dew or frost either at the ground or on the foliage of the hillside itself.

The values of the average potential temperature $\bar{\theta}$ below the first inversion, as computed from the 2 -hr averages of the temperatures, are plotted in cross section down the transect in Fig. 5 for two nights of the observation series. The scaling height used to calculate these values for stations I and IV are computed from the value for the mid-slope station III, the relative slope at these three stations, and their distance downslope from point $\mathrm{C}$ by relation (31) to be derived below.

There are two general types of variation, the 11 and 15 June (Fig. 5) being typical of each, respectively. On

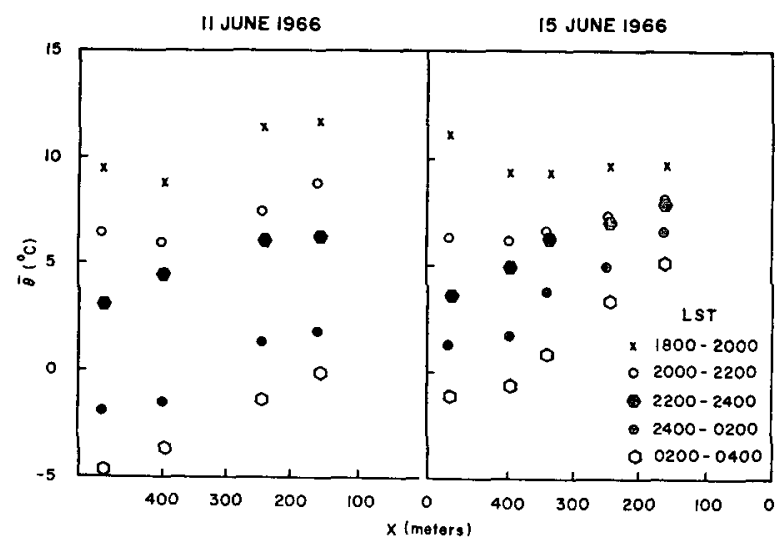

FIG. 5. Variation of $\bar{\theta}$ with downslope distance, 11 and 15 June 1966. 


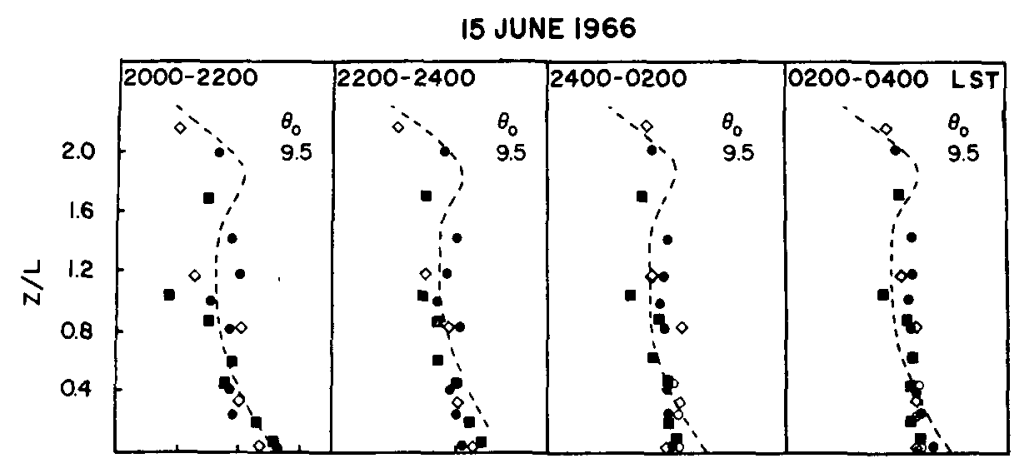

16 JUNE 1966

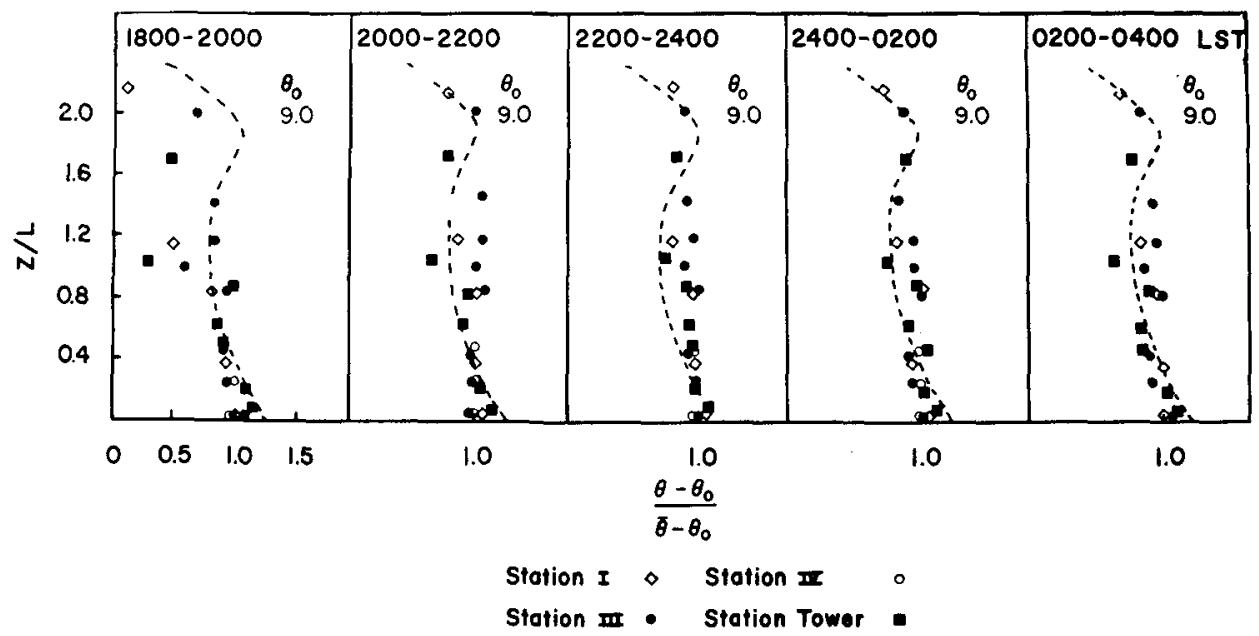

FiG. 6. Composite profiles of potential temperature deficit, 15 and 16 June 1966.

the former date, the temperature drop along the slope tended to remain constant through the night as indicated by the lines of best fit being almost parallel, while the temperature at the crest decreased with time through an interval almost five times the temperature drop down the slope. On the night of 15 June, on the other hand, the temperature at the crest remained essentially constant through the night, while the total slope gradient showed more than a tenfold increase. An examination of the transect profiles for the other nights shows a succession of the two types of variation, with the crest temperature tending to reach a nearly constant value in the early morning while the slope gradients depart from a nearly constant value in the evening to show a steady increase through the morning.

Although the relative values of $\bar{\theta}$ at the various stations show irregular variations through the night, in general, the values fall linearly downslope along the transect, particularly between the origin, point $\mathrm{C}$, and the tower.

Much of the following development will be based on the assumption that the vertical profiles of the potential temperature deficit, defined as the difference between the potential temperature $\theta$ at a point in the drainage flow and the potential temperature $\theta_{0}$ above the flow at that location, as computed from the measured temperature profiles at the different stations on the slope, are similar, i.e.,

$$
\frac{\theta_{0}-\theta}{\theta_{0}-\bar{\theta}}=\text { function }(z / L)=f_{\theta}(Z / L)
$$

The function $f_{\theta}$ will be further assumed to be invariant between the intervals of observation, with the exception of minor changes between the nights of 11 and 12 June 1966, and the remaining observations.

To establish the plausibility of these two assumptions, a set of composite plots of $\left(\theta-\theta_{00}\right) /\left(\bar{\theta}-\theta_{00}\right)$ vs $(z / L)$, where $\theta_{00}$ is the temperature at $\mathrm{C}$, were made for the various stations and 2-hr periods of the 1966 observations. Some typical examples are given in Fig. 6.

If it is assumed that the atmosphere above the drainage flow is adiabatic, then $\theta_{0}$ is equal to the crest temperature $\theta_{00}$ as obtained by a linear extrapolation along the transect plot of $\bar{\theta}$ for the periods involved. If there is an exterior lapse rate, the major effect would be to displace the plots of $\left(\theta-\theta_{0}\right) /\left(\tilde{\theta}-\theta_{0}\right)$ toward higher values relative to those shown, the effect being most marked at the tower station. 
14 JUNE 1966

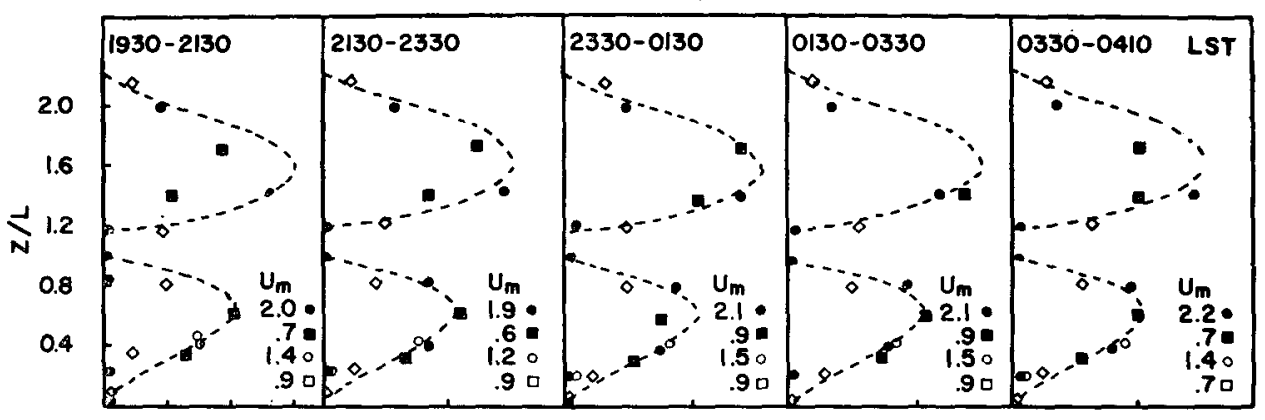

15 JUNE 1966

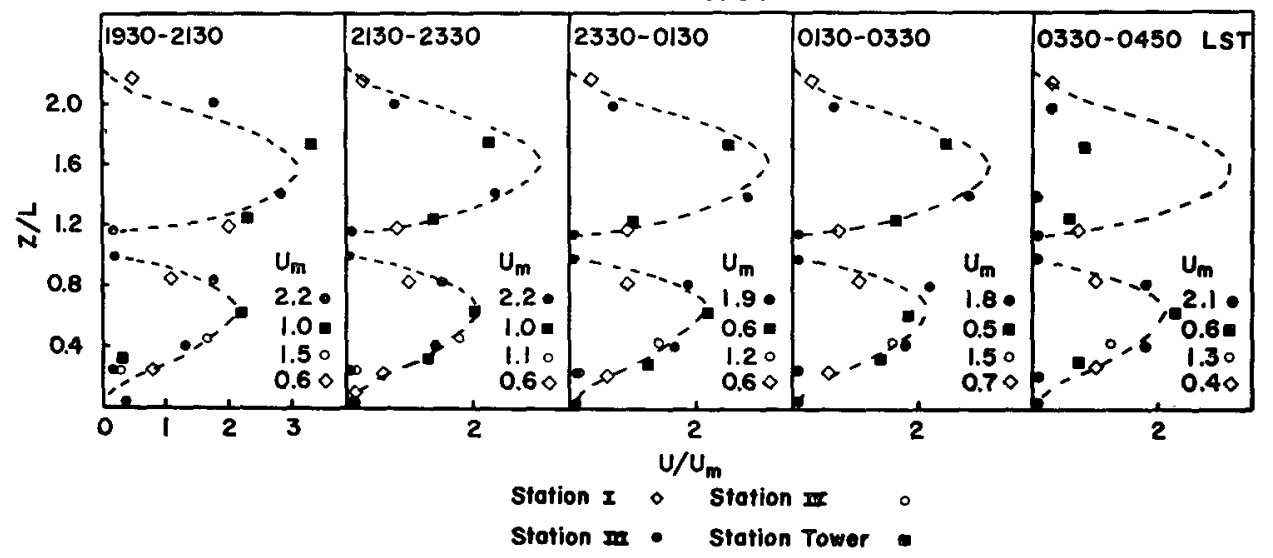

FIG. 7. Composite profiles of wind speed, 14 and 15 June 1966.

In evaluating these plots, it should be noted that the nominal error of temperature measurement, about $0.2 \mathrm{C}$, will amount to a much larger deviation in $\left(\theta-\theta_{0}\right) /$ $\left(\bar{\theta}-\theta_{0}\right)$ at the top of the transect than at the bottom. Thus, for a total slope drop of $5 \mathrm{C}$, the errors would be $20 \%$ at the upper station and less than $5 \%$ at the tower.

The dashed lines drawn over the points in Fig. 6 are polynomial segments computed to yield plausible boundary conditions for a second inversion at $(z / L)=3.0$, i.e. as determined from (8) below, and observed average values at the inversion and at the slope surface.

For the nights of 11 and 12 June, the associated wind profile at the mid-slope station indicated that the height of the velocity maximum had decreased, compared to the remaining nights of observation, together with a downward drift of the region of low temperature gradient below the temperature maximum; the latter, however, showed no significant displacement. Taken together, this seemed to indicate a reduction of the scale for the lower and upper flows and relative extension of the region of very low wind speeds. The dashed profiles for these nights differ from those of the remaining nights of 1966 since the intermediate region represented by a straight line segment in the previous profiles has been extended. For the purpose of this paper, the polynomial segments need to be regarded only as empirical summations of the data. On this basis, the points stay within about $10 \%$ of the same profile for most of the observation periods and levels in accord with the last assumption above, and they superimpose to a good approximation for most of the intervals and levels.

\section{b. Wind speed}

The vertical profile for wind speed on the slope over a 2-hr interval is illustrated in Fig. 4 for the same period as that used for the temperature profile. In contrast to the rather weak vertical gradients of the temperature profile, the wind speed shows strong vertical shears.

The flow seems centered about the inversion level with maximum near 5 and $12 \mathrm{~m}$ height separated by a region of almost vanishing velocity near the first inversion level $L$. Speeds in this last area vary from about 5-20 $\mathrm{cm} \mathrm{sec}^{-1}$ as contrasted with $1-5 \mathrm{~m} \mathrm{sec}^{-1}$ at the maxima. Like the temperature inversion, the pattern is persistent and found with slight variation in each profile of 10-min average speed.

Composite profiles of the velocity at the various stations were computed, as was done for the potential temperature deficit. The results for two nights are shown in Fig. 7. The assumption is analogous to that made for the $\theta$ profiles, i.e., $U / U_{m}=$ function of $(z / L)$, where $U_{m}$ is the mean value of the flow speed beneath level 1 . The scaling velocity was computed by the trapezoidal rule for the levels of measurement. The intervals of observation are not spaced as regularly as for the temperature profiles because of the various problems involved in testing the equipment. As the error in the wind speed measurements tends to be a constant percentage with the thermistor anemometers, the deviations for the various stations in Fig. 7 should be equally weighted.

Considering the strong shears just below the inversion level, it seems apparent that the observed velocity pro- 
file will be far more sensitive to scale changes than the temperature profile. Thus, the need for a modification of the height scale mentioned above was mainly apparent from the reduction in speed at the $6 \mathrm{~m}$ level at the mid-slope station for the nights of 11 and 12 June, and was supported, although less dramatically, by the variations at the other stations.

The dashed lines are cubic functions analogous to those used for the temperature-deficit profiles. The inversion region is assumed to be effectively motionless.

In the lowest region, the fit to the speeds on the steeper portions of the slope, that is, at station III, is not good and it is apparent that the similarity assumption is implausible in these levels.

Among the more obvious deviations from the typical profile of Fig. 4 is persistent separation at the mid-slope station for the major part of the night of 15 June and the first profile of 16 June. Such separation is implied by the increase of speed from the $1.5 \mathrm{~m}$ level to the 0.3 $\mathrm{m}$ level, which indicates back flow near the slope surface. Since the low-level shear and temperature gradient do not vary markedly from these intervals to others without such effects, it would appear that the mid-slope flow is on the verge of separation during all the periods of observation.

\section{Similarity analysis}

In the following development, the stand of trees on the experimental slope will be approximated by a hypothetical regular configuration of solid elements of equal basal area. The drainage flow on the slope will be considered to consist of a large-scale continuous motion through numerous identical and uniform channels of width $D$ corresponding to the interconnecting inter-tree spaces as depicted in Fig. 8. It will be assumed that 1) $D$ does not vary with height; 2 ) there is no motion immediately behind or in front of a tree; 3 ) the measured vertical profiles of wind speed and air temperature refer to conditions at the midpoint of any such channel at that particular distance along the mean streamlines from the virtual origin of the flow at point $\mathrm{C}$ of Fig. 1;4) for a space extending along the mean streamlines of Fig. 1 for more than a few mean tree diameters, the midline velocity profile assumes a characteristic value which varies little between such spaces at a particular downslope distance from the vertical origin ; 5) canopy storage change, soil heat flux, and accelerations are negligible; 6$)$ the slope and foliage surfaces radiate as blackbodies at the mean air temperature of the drainage layer, and the sky back-radiation is constant over the slope; 7) the flow in the space between the trees is optically thin in regard to thermal radiation and unsaturated; 8) the fluxes of sensible heat and momentum by molecular diffusion are negligible by comparison with the corresponding turbulent fluxes, with the possible exception of a thin layer at the surface of the slope; 9) Coriolis effects are negligible; and 10) the potential tem- perature deficit corresponding to the foliage temperature is a constant multiple $\omega$ of the potential temperature deficit for the measured air temperature profile at the same level above the slope, which lies between unity and the ratio of the potential temperature deficit at the slope floor to that $1 \mathrm{~m}$ above the slope between trees (that is, 1.15).

The control volume and the associated coordinate systems are diagramed in Fig. 8.

On the basis of the above assumptions, the balance equations for momentum and sensible heat for a unit volume of the flow space may be written as

$$
\begin{aligned}
& 0=-u \frac{\delta u}{\delta x}-\frac{\delta u}{\delta z}+\frac{1 \delta \tau_{f}}{\rho \delta y}-\frac{1 \delta P}{\rho} \frac{\delta P}{\delta x}+g \sin \phi+\frac{1}{\rho} \frac{\partial \tau}{\partial z} \\
& 0=-u \frac{\delta \theta}{\delta x}-\frac{\delta \theta}{\delta z}-\left(\frac{1}{C_{p \rho}}\right) \frac{\delta h}{\delta z}-\left(\frac{1}{C_{p \rho}}\right) \frac{\delta h_{f}}{\delta y}, \\
& 0=\frac{\delta P}{\delta z^{\prime}}+g \rho .
\end{aligned}
$$

In the above expression, $u$, $w$ are the velocities along the downslope axis and normal to the slope, respectively, $\tau_{f}$ and $\tau$ the eddy shear stresses along the coordinate axes $Y$ and $Z$ in that order, and $h_{f}$ and $h$ the analogous fluxes of sensible heat, $\theta$ the potential temperature as

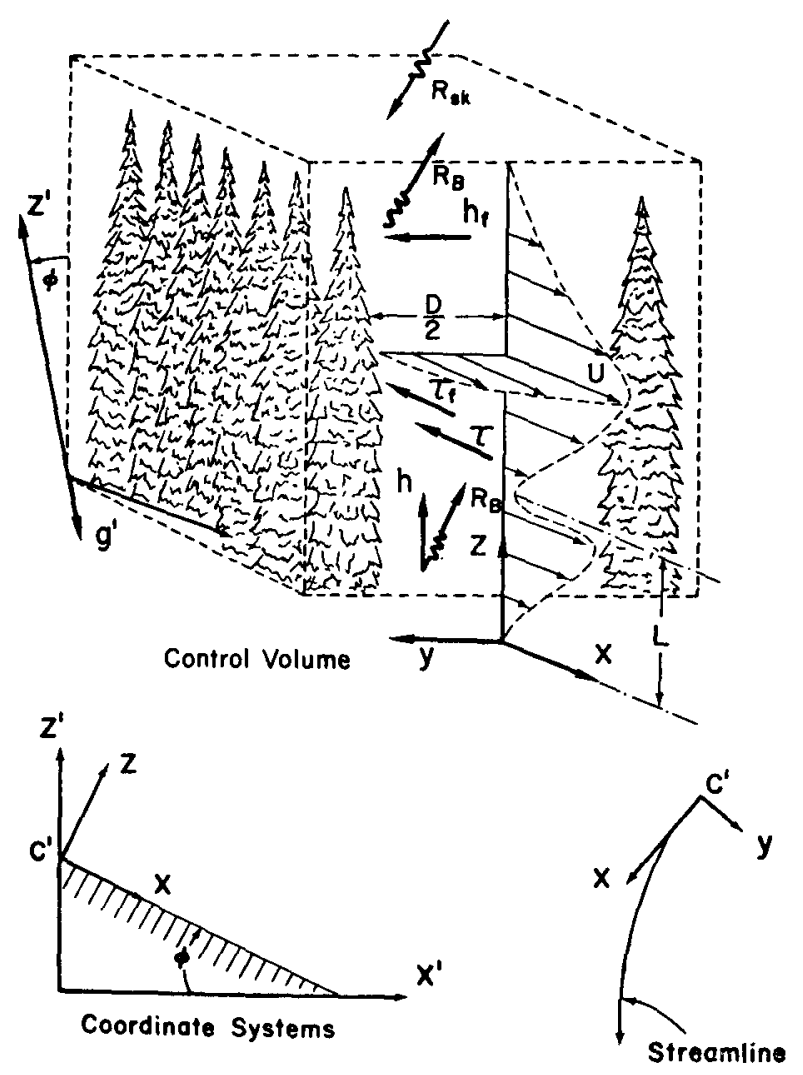

FIG. 8. Coordinate systems and control volume. 
defined above, $\rho$ the density of air, $C_{p}$ the specific heat of air at constant pressure $\left[0.240 \mathrm{cal} \mathrm{gm}^{-1}\left({ }^{\circ} \mathrm{K}\right)^{-1}\right]$, and $P$ the local pressure.

For continuity

$$
\frac{\delta w}{\delta z}+\frac{\delta u}{\delta x}=\frac{-1}{A^{\prime \prime}} \frac{d A^{\prime \prime}}{d t} .
$$

Assuming

$$
\frac{1}{A^{\prime \prime}} \frac{d A^{\prime \prime}}{d t}=\stackrel{\kappa}{x}=-
$$

where $\kappa$ is a constant depending on topography, and integrating the resultant form of (4) yields

$$
w=\int_{0}^{z}-\frac{u \kappa}{x}-\frac{\delta u}{\delta x} d z
$$

The potential temperature above the drainage flow layer is assumed to vary as

$$
\theta_{0}=A^{\prime} z+B x+\theta_{00}
$$

where $A^{\prime}$, the lapse rate normal to the slope surface, is small relative to the average vertical temperature gradient in the drainage layer.

The solutions to be examined of (1)-(6) for $\theta$ will be of the form

$$
\theta-\theta_{0}=\theta_{m} f_{\theta} h_{\theta},
$$

where $\theta_{m}$ varies only with $x$. The factor $f_{\theta}$ is a function only of the scaled height $(z / L)$ denoted as $\eta$. The factor $h_{\theta}$ is a function only of the scaled distance of the point from the center of the inter-tree space $(2 y / D)$ denoted as $\xi$.

The boundary conditions required of $f_{\theta}$ are

$$
f_{\theta}=\dot{f}_{\theta}=0 \text { at } \eta=\eta_{*},
$$

where the dot indicates differentiation by the transformation variable, $(\eta)$ in this case, and where $\eta_{*}$ is the scaled height of the second inversion above the slope. The analogous conditions on $h_{\theta}$ are

$$
h_{\theta}=\omega \text { at } \xi= \pm 1 \text { and } h_{\theta}=1 \text { at } \xi=0 .
$$

The associated drainage velocity solution will be

where

$$
U=U_{m} f_{u} h_{u},
$$

$f_{u}=0$ at $\eta=0$, and at $\eta=\eta_{*}, \quad \dot{f}_{u}=0$ at $\eta=\eta_{*}$,

and where

$$
h_{u}=0 \text { at } \xi= \pm 1 \text {, and } h_{u}=1 \text { at } \xi=0 .
$$

The relative change in local pressure is assumed to be small compared to the relative temperature change throughout the night, and the air is presumed to behave as an ideal gas with an effectively constant coefficient of thermal expansion.
The vertical velocity may be readily eliminated from (1) and (2) by (5). On substitution of the transformed variables, elimination of the pressure gradient by (3), integration over the inter-tree space, i.e., from $\xi=+1$ to $\xi=-1$, and neglecting a term small by (6), the momentum and sensible heat balance equations become

$$
\begin{aligned}
0=-U_{m} \frac{d U_{m}}{d x} f_{u}{ }^{2} & +\frac{U_{m}}{L}\left[\frac{\kappa U_{m} L}{x}+\frac{d\left(U_{m} L\right)}{d x}\right] \\
& \times \dot{f}_{u} \int_{0}^{\eta} f_{u} d \eta+g \alpha \gamma \theta_{m} f_{\theta} \frac{\lambda_{3}}{\lambda_{\mathbf{1}}}+\frac{2}{\rho \tau_{f 0}} \\
& +\frac{1 \delta \bar{\tau} \lambda_{1} D}{\rho} \frac{1}{\delta z}+g \alpha \cos \phi-\frac{\left.\theta_{m} L\right)}{d x} \frac{\lambda_{3}}{\lambda_{1}} \\
& \times \int_{\eta}^{\infty} f_{\theta} d \eta+g \alpha \cos \phi \theta_{m} \frac{d L}{d x} \frac{\lambda_{3}}{\lambda_{1}} f_{\theta}
\end{aligned}
$$

$$
\begin{aligned}
& 0=U_{m} \frac{d \theta_{m}}{d x} f_{u} f_{\theta}-\frac{\theta_{m}}{L}\left(\frac{U_{m} L_{\kappa}}{x}+\frac{d\left(U_{m} L\right)}{d x}\right) \\
& \times \dot{f}_{\theta} \int_{0}^{\eta} \hat{f}_{u} d_{\eta}-\frac{2 h_{f 0}}{D C_{p} \rho \lambda_{2}}-\frac{1}{C_{p} \rho \lambda_{2}} \frac{\delta \bar{h}}{\delta z} \\
& -B U_{m} \stackrel{\lambda_{4}}{-} f_{\lambda_{2}}-A^{\prime} U_{m} \frac{d L}{d x} \frac{\lambda_{4}}{\lambda_{2}} f_{u} \eta,
\end{aligned}
$$

respectively, where

$$
\left.\begin{array}{rl}
\lambda_{1} & =\frac{1}{2} \int_{-1}^{+1} h_{u}^{2} d \xi \\
\lambda_{2} & =\frac{1}{2} \int_{-1}^{+1} h_{\theta} h_{u} d \xi \\
\lambda_{3} & =\frac{1}{2} \int_{-1}^{+1} h_{\theta} d \xi \\
\lambda_{4} & =\frac{1}{2} \int_{-1}^{+1} h_{\theta} d \xi \\
\gamma & =\sin \phi \\
\alpha & =\frac{1}{\theta_{0}}
\end{array}\right\}
$$

Since the lateral transport of sensible heat and momentum involves no direct work against buoyancy forces, it seems plausible to assume a turbulent Prandt1 number of unity for such transport of the form

$$
\tau_{f 0}=-\frac{h_{f 0}}{C_{p}}\left[\frac{\delta u}{\delta y}\left(\frac{\delta \theta}{\delta y}\right)^{-1}\right]_{y= \pm D} .
$$


By the previous discussion, the net radiation loss from the foliage may be expressed as

$$
R=\frac{R_{0} E}{\eta L}
$$

where $E$ is a constant depending on the distribution of foliage surface, previously estimated as 0.05 for the experimental slope. If the sensible heat flux to the foliage is to balance this loss,

$$
h_{f 0}=\frac{1}{2 \epsilon^{\prime}} \frac{R_{0}}{\eta} \frac{E}{L}, \text { where } \epsilon^{\prime}=\frac{\epsilon}{1-\epsilon} \cong \epsilon .
$$

The vertical eddy flux of sensible heat and momentum may be formally expressed in terms of an eddy conductivity $K_{h}{ }^{\prime}$ and eddy viscosity $K_{m}{ }^{\prime}$, although no presumptions may be made concerning the variation of these parameters. When the corresponding substitutions are made in (13) and (14), it is found that analytical similarity of the velocity and of the potential temperature deficit profiles requires that:

$$
\begin{aligned}
& \left(\frac{A^{\prime} U_{m}^{2}}{\theta_{m}^{2}}\right) \frac{d L}{d x} \frac{\lambda_{4}}{\lambda_{2}} \frac{\lambda_{1}}{\lambda_{3} g \alpha \gamma}=\rho, \\
& \left(\frac{\lambda_{1} U_{m}}{\lambda_{3} g \alpha \gamma \theta_{m}}\right) \frac{d U_{m}}{d x}=a, \\
& \frac{\lambda_{1} U_{m}}{L \lambda_{3} g \alpha \gamma \theta_{m}}\left[\frac{U_{m} L_{k}}{x}+\frac{d\left(U_{m} L\right)}{d x}\right]=d \text {, } \\
& \left(\frac{R_{0} E}{D \epsilon C_{p}}\right)\left(\frac{U_{m}}{g \rho \alpha \gamma L \theta_{m}{ }^{2} \lambda_{3}}\right)^{\dot{h}_{u}(1)}{\dot{h_{\theta}}(1)}_{1}=c, \\
& \left(\frac{\lambda_{\mathbf{1}} \lambda_{4} U_{m}}{\lambda_{3} L^{2} \theta_{m} g \alpha \gamma}\right) \frac{d\left(\bar{K}_{m}^{\prime} \dot{f}_{u}\right)}{d \eta} \neq j \ln (x)=n, \\
& \frac{\cos \phi}{\gamma} \frac{d L}{d x}=l, \\
& \left(\frac{\lambda_{1} U_{m}^{2}}{g \gamma \theta_{m}^{2} \alpha \lambda_{3}}\right) \frac{d \theta_{m}}{d x}=e, \\
& \frac{\lambda_{1} E R_{0} U_{m}}{\lambda_{3} \lambda_{2} D C_{p} \rho \gamma g \alpha \theta_{m}^{2} L \epsilon^{\prime}}=b, \\
& \frac{\lambda_{4} B \lambda_{1} U_{m}^{2}}{g \lambda_{2} \lambda_{3} \alpha \gamma \theta_{m}^{2}}=f, \\
& \left(\frac{\lambda_{1}}{\theta_{m} \lambda_{2} g \alpha \gamma L^{2}}\right) \frac{d\left(\bar{K}_{h}^{\prime} \dot{f}_{\theta}\right)}{d \eta} \neq j \ln (x)=i \text {, } \\
& \frac{\cos \phi}{\gamma \theta_{m}} \frac{d\left(\theta_{m} L\right)}{d x}=m,
\end{aligned}
$$

with the "similarity constants" $a, c, l, e, b, f$ and $\rho$. In (22) and (27), $n$ and $i$ may be functions of $\eta$ since we have not explicitly stated the structure of $K_{h}{ }^{\prime}$ and $K_{m}{ }^{\prime}$.

If the analysis of the problem is confined to the portion of the slope where the potential temperature drop may be reasonably approximated by

$$
\theta_{m}=A x,
$$

an immediate result by (24) is

$$
U_{m}=\left(\frac{A \operatorname{erg\alpha \lambda _{3}}}{\lambda_{1}}\right)^{\frac{1}{2}} x
$$

The sensible heat balance may be integrated over the layer $\eta=0$ to $\eta=\infty$ with the lower bound being approximated by some small increment, $\sim 1 \mathrm{~cm}$. In performing the integration, the integral of the eddy flux of sensible heat to the foliage and to the ground surface is assumed to equal the net radiation loss from the slope per unit area of inter-tree flow space: $R_{0} / \epsilon$. The result, using (29) and (30), and for the case where $B / A$ is negligible, is

$$
L=\frac{R_{0} x}{U_{m} \theta_{m} \epsilon(\kappa+1) \lambda_{2} r_{1} C_{p \rho} \rho}
$$

where

$$
r_{1}=\int_{0}^{\infty} f_{u} f_{\theta} d \eta
$$

The effect of an appreciable value of $B / A$ is to decrease the depth of the flow. An immediate inference from this integration is that $L$ varies as $1 / x$ in both cases, thus the flow may exist only over a finite part of any uniform slope, which is consonant with the choice of (29).

Eqs. (30) and (31) indicate that (29) satisfies (20), (21) and (25). Eqs. (23) and (18) are associated with terms which vary as $1 / x^{2}$ compared to $1 / x$ for the remaining advective terms of the momentum and sensible heat balance, and which for points more than a few meters from the origin should be negligible.

Eq. (19) in combination with (29) and (30) requires either that $\gamma$ varies as a power of $x$, or that the relative downslope rate of change of $\gamma$ be small relative to the value of $\gamma$ at any point, a condition generally met on the experimental slope.

The number of independent similarity constants may now be reduced by the relations: $a=e ; e=\kappa d$; $b=(2 E / D) r_{1}(\kappa+1) e ; c=2(E / D) r_{1}(\kappa+1) e$.

While the vertical eddy exchange coefficients have not been explicitly formulated, (22) and (27) are seen to imply that both are proportional to $x^{-2}$.

Integration of the scaled momentum equation from $\eta=0$ to $\eta=\infty$ yields a solution for the similarity constant $e$. In evaluating the surface stress terms, the derivatives $\dot{f}_{u}(0), \dot{f}_{\theta}(0)$ are evaluated as finite differences at 
14 JUNE 1966

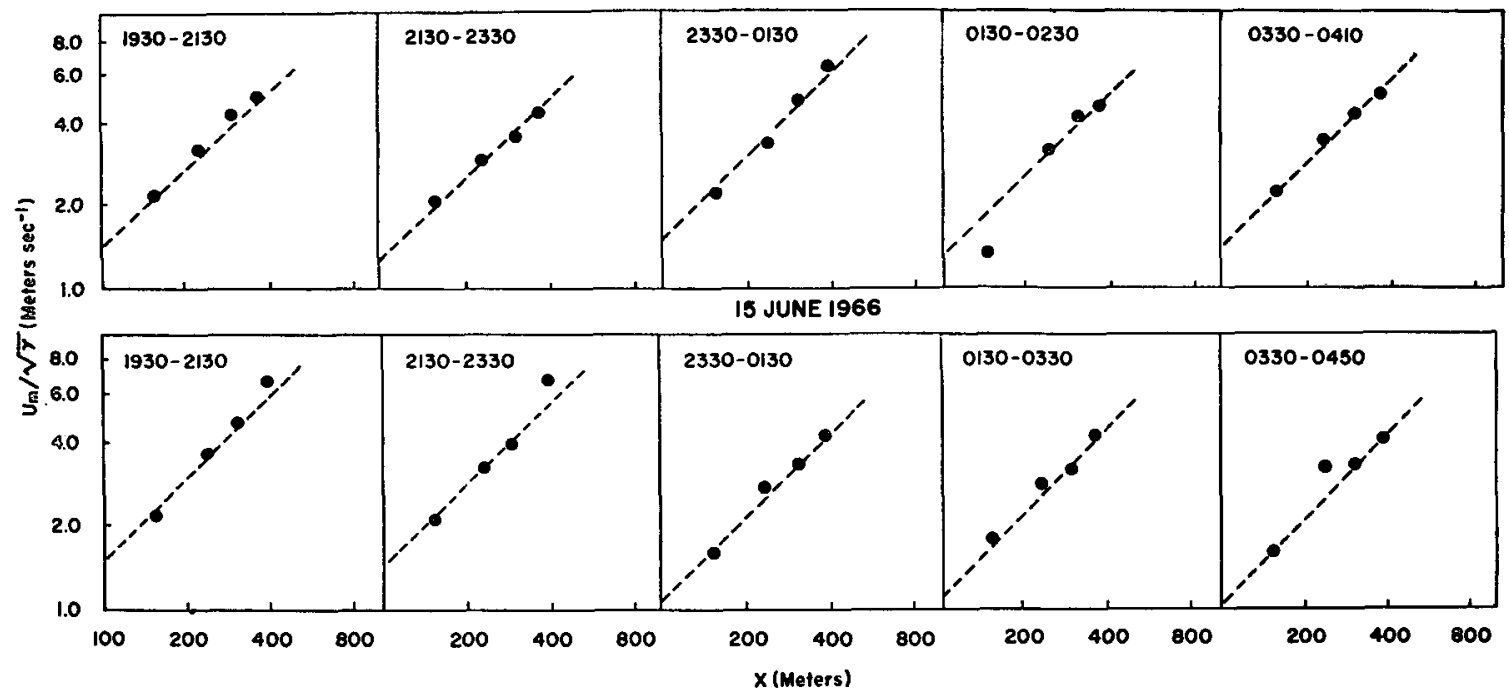

FIG. 9. Variation of $U_{m} / \sqrt{\gamma}$ with downslope distance, 14 and 15 June 1966.

$\eta=0.2$ at which level the advective terms are small enough to neglect, judging from the observed profiles.

For the case where $B / A$ is small:

$$
\begin{aligned}
e=\left[\frac{r_{3}}{r_{1}(\kappa+1)}\right]\left[\frac{r_{6}}{r_{1}}-\left(\frac{2 \beta C^{\prime} \epsilon \lambda_{5}}{\lambda_{1}}\right)\right. & r_{5} \lambda_{2} \\
& \left.-\frac{E}{D} \gamma_{4} \frac{\lambda_{2}}{\lambda_{1}}\left(\frac{h_{u}(1)}{h_{\theta}(1)}\right)\right]^{-1},
\end{aligned}
$$

where $\beta$ is the Prandtl number near the slope surface, and where
(a) $r_{6}=\int_{0}^{\infty} f_{u}^{2} d \eta$
(b) $\left.r_{5}=\frac{-f_{u}(0.2)}{f_{\theta}(0.2)-f_{\theta}(0)}\right)$
(c) $r_{4}=\int_{0}^{\infty} \frac{f_{u}}{\eta f_{\theta}} d \eta$
(d) $r_{3}=\int_{0}^{\infty} f_{\theta} d \eta$
(e) $\lambda_{5}=\int_{0}^{1} \frac{h_{u}}{h_{\theta}} d \xi$

where $f_{u}$ approaches zero rapidly enough as $\eta \rightarrow 0$ to avoid problems from the reciprocal term in the integral $\boldsymbol{r}_{4}$.

If invariance of the height scale with total radiation loss is assumed for cases where $B / A$ is small, then (29), (30) and (31) imply that

$$
A \propto R_{0}{ }^{3} .
$$

For this result, the similarity groupings considered with the exception of $i$ and $n$ are obviously independent of $R_{0}$. The Richardson's number for the flow, including the lateral stress terms, shows no direct dependence on $A$ and thus $R_{0}$. If the turbulent intensity is assumed pro- portional to the local shear, the eddy flux terms $(i, n)$ should show no direct dependence on $R$.

In summary, the observed profile forms $f_{\theta}, f_{u}$, for an adiabatic external condition should be independent of the net radiation rate, and thus, of the slope temperature drop and drainage speed. The situation in regard to the foliage parameters is not as clear. The ratio $E / D$ would not be conservative since an increase in $E$ would normally imply a decrease in the average tree spacing. Dependence upon the topographic term $\kappa$ is obvious for all the similarity constants except $(a)$ and $(e)$.

Without a formulation of the eddy exchange coeffcient, it is difficult to state on the basis of the analysis what relevance the observed profile forms of potential temperature deficit and velocity on the experimental slope would have to flows on terrain of different configuration and forest cover.

\section{Analysis of data}

The downslope variation of $U_{m} / \sqrt{\gamma}$ along the transect is shown in Fig. 9 for the periods of the 1966 observations, for which the vertical profiles were given in Fig. 7 . The number of data points available varies due to malfunctions at station IV on some nights.

Deviations from the linear variation predicted by (30) tend to vary systematically with the constancy of the temperature at the virtual origin $\mathrm{C}$ of Fig. 1. Periods with decreasing temperatures at $\mathrm{C}$, as on the night of 11 June at station III, which is on the steepest section of the transect, show an excess of local wind speed relative to the rest of the stations. The deviations from linearity are in the reverse sense for periods of relatively constant temperatures at point $\mathrm{C}$. Large deviations are not apparently associated with separation of the flow, but are associated with periods of strong midslope acceleration. On the whole, the plots tend to support (30). 


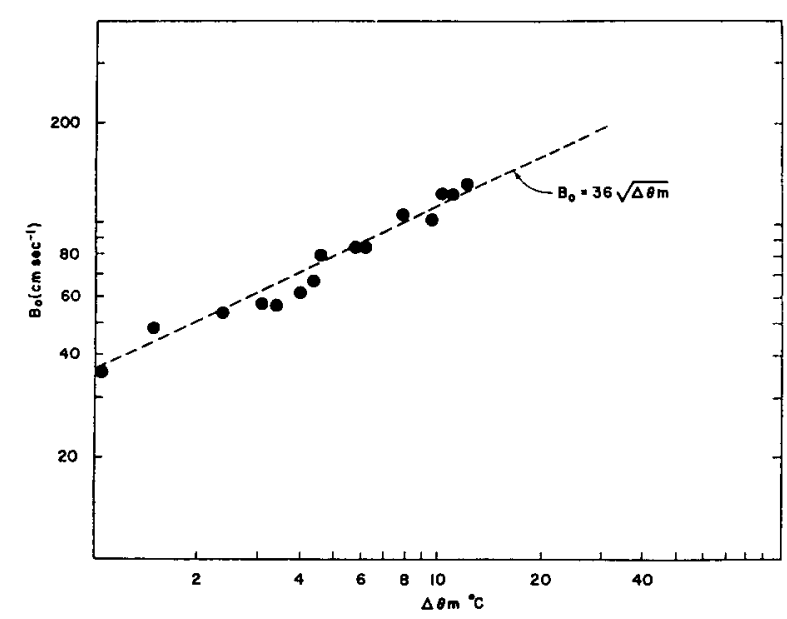

FIG. 10. Variation of velocity intercept with potential temperature drop down the slope.

The values of the intercept at $x=100 \mathrm{~m}$ (denoted $B_{0}$ ) of the fitted line were estimated from similar plots for all the 1966 observations. The midslope averages of 1964 and the base averages of 1962 were used to estimate the corresponding $B_{0}$ values by assuming a linear variation as was found plausible for the 1966 data.

If there is a significant potential temperature drop $\Delta \theta_{0}$ down the slope outside the drainage flow layer, then the constant $A$ in (29) must be proportional to the difference $\Delta \theta-\Delta \theta_{0}$ rather than to $\Delta \theta_{0}$. The temperature drop down the hill just before the onset of drainage, at 1800 , was used as an estimate of $\Delta \theta_{0}$.

A plot of $B_{0}$ vs $\Delta \theta$, or in three cases of $\Delta \theta-\Delta \theta_{0}$, is shown in Fig. 10. The line of best fit, as graphically estimated, has a slope of about 0.5 , which agrees with (30) above. The intercept yields a calculated value of $\left(\gamma e \alpha g \gamma_{3} / \gamma_{1}\right)^{\frac{1}{2}}=0.7$ with $\Delta \theta$ calculated for a downslope distance of $390 \mathrm{~m}$ from $\mathrm{C}$.

The profile integrals of (31) and (34) were computed graphically from the composite profiles. The extension of the central low velocity zone for the nights of 11 and 12 June has no effect on $\boldsymbol{r}_{6}, \boldsymbol{r}_{1}$ and $\boldsymbol{r}_{2}$, and only increases $r_{4}$ and $r_{3}$ by about $10 \%$; thus, no separate calculation was made for these nights; $f_{u} / f_{\theta}$ falls so rapidly near the surface that the evaluation of the reciprocal term posed no problem in practice. Similarly, the decrease of $f_{u}$ near the top of the canopy $(\eta>2)$ was such that these regions contributed less than $10 \%$ of the total integral through the drainage layer, which is fortunate in view of the inaccuracy of the power law representation of the foliage surface as shown in Fig. 5 for these heights.

The profile integrals are calculated to be: $r_{1}=1.5$, $r_{2}=1.5, r_{3}=1.0, r_{4}=2.1$, and $r_{6}=3.2$.

The evaluation $r_{5}$ defined in (34b) is not a straightforward matter. In the region below $\eta=0.2$, the superposition of the station profiles is quite imperfect. The value of $f_{u}(0.2)$ for the midslope stations is no greater than 0.1 , and in view of the separation observed, very likely far less than this.
The appropriate value for the stations on the milder slopes seems to be 0.3 . Thus, the value adopted, 0.25 , is in the nature of an upper bound. Since $f_{\theta}(0)-f_{\theta}(0.2)$ is $0.1, r_{5}=2.5$.

The parameters depending on the distribution of foliage on the slope have been estimated as: $\epsilon=0.18$, $C^{\prime}=0.6, D=100 \mathrm{~cm}, E=0.05$.

The values of $\lambda_{1}, \lambda_{2}, \lambda_{3}, \lambda_{4}$ and $\dot{h}_{u}(1) / \dot{h}_{\theta}(1)$ must for lack of horizontal profile data for wind speed and temperature be estimated. A parabolic variation of the form

$$
\begin{aligned}
& h_{\theta}=1+(\omega-1) \xi^{2}, \\
& h_{u}=1-\xi^{2},
\end{aligned}
$$

was assumed, and evaluation of the defining integrals by the functions, with $\omega=1.15$, yielded the values 0.5 , $1.06,1.05,0.7,1.0$, for $\lambda_{1}, \lambda_{2}, \lambda_{3}, \lambda_{4}, \lambda_{5}$, respectively. The value of $h_{u}(1) / \dot{h}_{\theta}(1)$ was computed as -6.7 . The parameter $\kappa$ has been shown to be near unity.

When the terms in the denominator of the expression for $e$ given in (33) are evaluated with these values, and assuming that $\beta=2.0$, the foliage-dependent term is found to contribute less than $2 \%$ as compared to the surface stress term contribution of about $25 \%$. The remaining and principal term depends only on the vertical profile integrals of (34). Thus, while the vertical profile integral functions are strongly dependent upon the foliage parameters, as shown above, the value of $e$ can be determined to a fair approximation without an accurate independent estimate of those parameters, given these profile functions. The value of $e$ is also relatively independent of the transverse profile constants. The value for the intercept of Fig. 10 computed from (29), using an assumed value for $g$ of $980 \mathrm{~cm} \mathrm{sec}^{-2}$, and an ambient temperature of $300 \mathrm{~K}$, is $42 \mathrm{~cm} \mathrm{sec}^{-1}$. The value estimated from the data is $36 \mathrm{~cm} \mathrm{sec}^{-1}$.

The appropriate net radiation loss for the individual periods was calculated from the output of the hemispherical radiometer and the slope temperature profile. The $R_{0}$ value was computed from the 2 -hr average of the effective sky radiation $R_{s k}$ and the 2 -hr average of the average slope temperature $T_{m}$ estimated from the temperature variation down the transect. The value of $R_{s i}$ was computed from the average radiometer plate output and the average plate temperature for that period. In 1964, such estimates were supplemented by actual measurements of the radiation loss at the midslope station with an unventilated net radiometer suspended over the canopy. Agreement between estimated and measured values was found to be within $20 \%$ (Bergen, 1969).

Fig. 11 is a plot of $\Delta \theta$ vs $R_{0}$ for all the periods except the rather anomalous 11 June observations and the 1962 periods for which no radiometer data were available. A line was drawn through the data with a slope of 0.7 , in agreement with (35). The data for 11 June indicates much smaller slope temperature drops for a given radiation loss. It would seem that if some exterior tempera- 
ture drop was responsible for this effect, it would have had to amount to a decrease of $\theta_{0}$ with height on the slope.

The intercept of the line through the points is $44 \mathrm{C}$ at $R_{0}=1 \mathrm{ly} \mathrm{min}^{-1}$. Using the observed inversion height and vertical profiles at station III with the value of $\left(e g \lambda_{3} / \lambda_{1}\right)$ obtained above, the intercept computed from (29) and (31) is 36C, which, considering that the calculation involves a crude approximation of $\epsilon$, is in reasonable agreement.

Since this calculation was initially presented, it has been pointed out to the author that, in view of the assumption of fully turbulent flow in the inter-tree spaces, the one-seventh power law for the velocity and temperature profiles across the inter-tree space would be a more appropriate assumption. When such an assumption is made, the profile functions above become: 0.40 , $0.97,1.13,0.88,0.97$ and -6.7 , respectively. These revised and probably more appropriate values introduce no significant change in the calculated value of the intercepts.

\section{Principal conclusions}

The measurements indicate that the vertical profiles of velocity and potential temperature at different locations on the slope and at different times at the same location have definite similarity properties. These properties have been exploited in a semi-empirical approach to the problem of relating the velocity distribution and temperature variation on the slope to the average net radiation loss.

For the hillside studied, it appears that the constant radiation similarity solutions for the mean flow and temperature deficit yield valid results for the observed flow. The downslope variation of the wind speed is in general agreement with the variation predicted by the solution and the slope of the hillside. The local mean speed varies as the one-half power of the temperature drop down the slope as predicted, and its magnitude is in reasonable agreement with that predicted by the solution, the estimated magnitudes of the foliage parameters, and integral functions of the observed vertical profiles of wind speed and potential temperature deficit.

The potential temperature drop down the hillside varies approximately as the two-thirds power of the estimated net radiation loss as predicted, and its magnitude is in fair agreement with that computed from the solution, the velocity constant, foliage distribution, and the observed inversion heights.

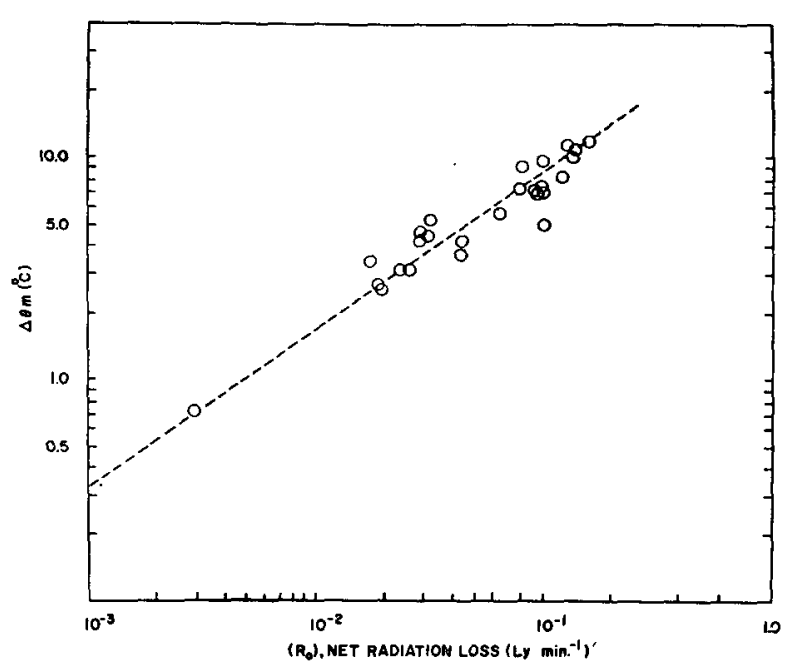

FIG. 11. Variation of slope temperature drop with net radiation loss.

The observed profile forms for potential temperature deficit and wind velocity appear to be substantially independent of net radiation loss over a considerable range of values, as predicted by the solution and the observed constancy of the inversion heights.

The model predicts a decreasing depth of flow for some distance downslope from the virtual origin of the flow. This virtual origin appears to coincide well with the divergence center located by a streamline analysis of the height contour map, assuming that the direction of flow is normal to the contours.

It appears probable that the observed profile forms have relevance for the upper reaches of most of the highly divergent forested slopes found in the $3000 \mathrm{~m}$ altitude zone in the central Rocky Mountains.

\section{REFERENCES}

Bergen, James D., 1969: Nocturnal radiation loss estimates for a forest canopy. USDA Forest Service Res. Note RM-155, 4 pp.

- 1967 : Cold air drainage on a forested mountain slope. Ph.D. dissertation, Colorado State University, Fort Collins.

Ives, R. L., 1939: River fogs in Middle Park, Colorado. Bull. Amer. Meteor. Soc., 20, 415-417.

Reifsnyder, W. E., and H. W. Lull, 1963: Radiant energy in relation to forests. Bull. 1344, U. S. Forest Service, U. S. Dept. of Agriculture, $111 \mathrm{pp}$.

Storey, T. G., W. L. Fons and F. M. Sauer, 1955: Crown characteristics of several coniferous tree species. Interim Rept., USFSWP 416, Div. of Fire Research, U. S. Forest Service, $92 \mathrm{pp}$. 